\title{
Bioinformatics a Tool for Biosystematics Studies of Lagenaria siceraria (Mol.) Standl. Complex
}

\author{
Fortune 0. Awala*, Benjamin C. Ndukwu, Ikechukwu O. Agbagwa \\ Department of Plant Science \& Biotechnology, University of Port Harcourt, Port Harcourt, Nigeria \\ Email: ^ihunnah.fortune@gmail.com, benjamin.ndukwu@gmail.com, ikechukwu.agbagwa@uniport.edu.ng
}

How to cite this paper: Awala, F.O., Ndukwu, B.C. and Agbagwa, I.O. (2019) Bioinformatics a Tool for Biosystematics Studies of Lagenaria siceraria (Mol.) Standl. Complex. American Journal of Plant Sciences, 10, $1729-1748$.

https://doi.org/10.4236/ajps.2019.1010123

Received: May 29, 2019

Accepted: October 13, 2019

Published: October 16, 2019

Copyright $\odot 2019$ by author(s) and Scientific Research Publishing Inc. This work is licensed under the Creative Commons Attribution International License (CC BY 4.0).

http://creativecommons.org/licenses/by/4.0/

\begin{abstract}
Bioinformatics has been a major tool in the revolution of plant systematics in recent times. The diversity of fruit shapes in Lagenaria siceraria (Mol.) standl. species has been of great concern because of its fruit complexity. This study is based on the application of rubisco enzyme using $r b c L$ marker because of its conservativeness and its ability to discriminate below the specific level hence its usage to sequence the chloroplast genome with ABI, PRISM 377 DNA sequencer. The sequences obtained were viewed using MEGA X software and subsequently subjected to validation through National Center for Biotechnology Information (NCBI) using Nucleotide Basic Local Alignment Search Tool (BLAST N). The result obtained showed that all the sequences belong to Lagenaria siceraria (Mol.) standl. with percentages ranging from $95 \%$ to $100 \%$ for query cover sequences and $98 \%$ to $100 \%$ for identity sequences. From the taxonomic report obtained sequence A, C, G, H, J, $\mathrm{Q}$ has the highest hits of 44 on Lagenaria siceraria out of 109 total value, sequence $\mathrm{O}$ and $\mathrm{R}$ has the highest hits of 44 on Lagenaria siceraria out of the total value of 111 , sequence $\mathrm{V}$ has the highest hits of 44 on Lagenaria siceraria out of 119 total value and sequence $\mathrm{X}$ finally has the highest value hits of 44 on Lagenaria sceriania out of 105 total value, based on this report, phylogenetic tree was constructed to show the level of relatedness of the different fruit diversity of $L$. siceraria complex. This work therefore has aided in the molecular characterization of Lagenaria siceraria (Mol.) standl. landraces found in Nigeria.
\end{abstract}

\section{Keywords}

Bioinformatics, Biosystematics, Phylogenetic, Diversity, Lagenaria siceraria (Mol.) Standl. Complex 


\section{Introduction}

The species Lagenaria siceraria (Molina) Standl is a member of the Cucurbitaceae family, Cucurbitaceae subfamily and Benincaseae tribe [1]. The genus Lagenaria consists of five other wild species, namely Lagenaria breviflora (Benth)

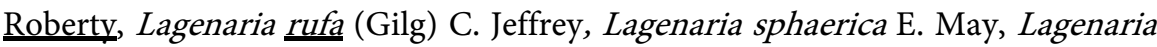
abyssinia (Hook F) C. Jeffrey, Lagenaria guinensis (G. Den) C. Jeffrey and Lagenaria siceraria (Molina) Standley is the most cultivated within the species of siceraria; two morphologically distinct sub-species of bottle gourd have been recognized thus Lagenaria siceraria subspecies asiatica and Lagenaria siceraria subspecies siceraria [2] [3] [4]. It is universally agreed fact, that different species are systems of population which exhibit variation and not fixed entities, therefore no individual is identical as developed by [5]. Systematics as defined by [6], states that systematics is a natural science that deals with the study of individual, population and taxon relationship for the purposes of classification. Plant systematics is therefore based on the premise that in the enormous variation in the plant kingdom there exist discrete units called species that can be identified classified and named with further logical relationships developed among these units. Over the years, plant groupings have been redefined as more information is accumulated from newer approaches and different recent sources such as molecular systematics, and taximetrics. Variety of Lagenaria is known throughout West Africa where they serve different economic purposes but these cultivars are largely unrecorded in terms of micromorphological characteristics [7] and diversity of fruit shape and sizes continue to create serious taxonomic difficulties in the delimitation of the taxon Lagenaria siceraria (Mol) Standley below the specific level.

The emerging field of molecular systematics deals with the exclusive utilization of molecular data [6]. A molecular marker is a gene or DNA sequence with a known location on a chromosome that can be used to identify species, which detect variation at the nuclear, Mitochondrial, and chloroplast DNA. Molecular markers have been developed and utilized to provide the requisite landmarks for clarification of genetic variation [8]. DNA is more stable macromolecule than RNA and is found in all plant tissues hence, DNA based markers are preferred for precise identification of plant species [9]. An understanding of the extent of genetic diversity within and amongst the landraces of the gourd is important for strategic breeding and conservation [10].

Plants are generally more tolerant of variation, hence genetic variation may result from mutation or recombination [6] [11]. According to [12], effective utilization of plant genetic resources depends on a detailed understanding of their genetic variability. Interestingly, substantial variation exists in the ability of plants to tolerate gene imbalance both between different plant species and between varieties of the same species [13]. However, despite the widespread interest in molecular systematics, there is limited understanding of the molecular mechanisms that lead to phenotypic alterations in plant species as well as gene interactions involved in the global genomic level [14], went further to explain 
that the molecular systematic studies give deeper insight into genetic structures. It has performed an important role in molecular biologies, such as analyzing genetic diversity by classification of cultivars and germplasm collections. It has also aided in clearing phylogenetic relationships among groups or closely related species. It is further used to determine the sequence of nucleotides in the DNA of plants, conclusively, has increased significantly, understanding of plant evolution.

\section{Materials and Methods}

\subsection{Study Map}

The species were planted in the faculty of Agricultural science research farm, of the University of Port Harcourt (Figure 1).

\subsection{Plant Material and DNA Isolation}

Twenty-four landraces of Lagenaria siceraria (Mol.) Standl. were collected from different locations of Nigeria and planted at Agricultural Science Research Farm. The young fresh leaves from A African Bottle Gourd, B Kettle Gourd, C Caveman Club Gourd, D Base Ball Gourd, F Bushel Gourd, G Bird House Gourd, H Water Jug Gourd, I Cup Gourd, K Warted Bushel Gourd, L Extra Large Pawpaw Gourd, M Long Siphon Gourd, N Indian Gourd, O Chinese Bottle Gourd, P Mini Dipper Gourd, Q Goose Neck Gourd, S Swan Gourd, T Palm Wine Gourd, U Long Handle Dipper Gourd, V Powder Horn Gourd, W Snake Gourd and X Microphone Gourd were analyzed at Biotechnology Research Centre of the University of Port Harcourt in Partnership with International Institute of Tropical Agriculture (IITA) Ibadan in 2018. The fresh leave sample from 21-day-old seedlings was crushed using liquid nitrogen. Genomic DNA was extracted using a Zymo Quick Plant/Seed extraction method (2010) and stored at $-20^{\circ} \mathrm{C}$. The quantity of the DNA was determined using Thermo Scientific ${ }^{\mathrm{TM}}$ NanoDrop 2000/2000C spectrophotometer, while the quality of DNA isolated was analyzed using $0.8 \%$ agarose gel electrophoresis and viewed with UV Transilluminator-BIOCOMdirect.

Polymerase chain reaction (PCR) analysis was carried out using standard PCR procedure. The amplification of the extracted DNA was done using Gene Amp PCR System 9700 Dual 384-well sample block module, according to the protocol of Applied Biosystems (2002). The rbcl primer having a forward sequence of F (5' ATGTCACCACAAACAGAAAC 3') and reverse rbcl primer sequence of $R$ (5' TCGCATGTACCTGCAGTAGC 3') with an expected size of $724 \mathrm{bp}, 10 \times$ PCR buffer 1.0, $50 \mathrm{mM} \mathrm{MgCl}_{2} 0.4,5 \mathrm{pMol}$ forward primer 0.5, $5 \mathrm{pMol}$ reverse primer 0.5, DMSO 0.8, 2.5 Mm DNTPs 0.8, Taq $5 \mathrm{u} / \mu \mathrm{l} 0.1,100 \mathrm{ng} / \mu \mathrm{l}$ DNA 3.0, $\mathrm{H}_{2} \mathrm{O} 2.9 \mu \mathrm{l}$ and a final volume of $10 \mu \mathrm{L}$. The amplification products were analyzed using a $1.5 \%$ agarose gel stained with EZ-Vision ${ }^{\circledR}$ in Gel solution. The samples are arranged A-X after the ladder and the ladder used is $50 \mathrm{bp}$ from NEB (Plate 1). 


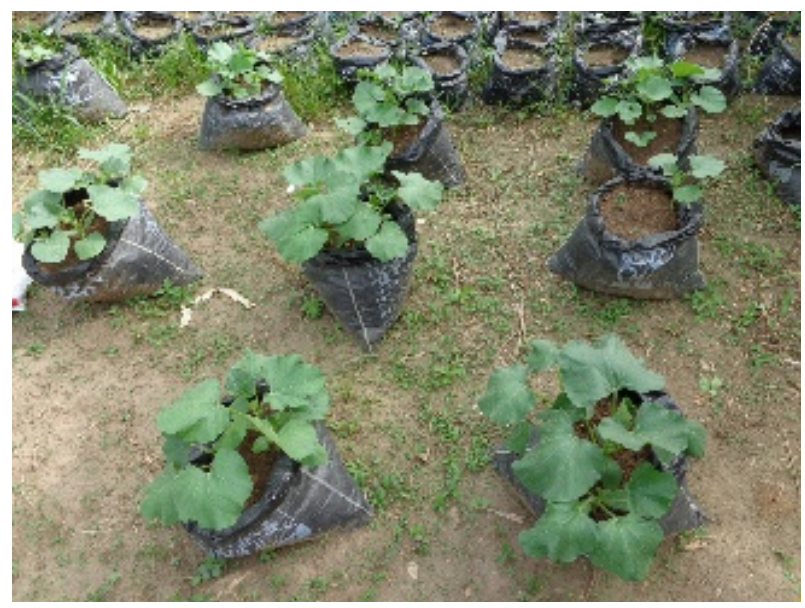

Plate 1. Showing 21 days old Lagenaria siceraria (Mol.) Standl.

The amplification products from land races of Lagenaria siceraria (Mol.) Standl. were separated using 1.5\% agarose electrophoresis and the expected size of bands were excised using scapel and purified by Spin Column Purification method. Purified amplicons were sequenced using BigDye Terminator v3.1 Cycle Sequencing Kit protocol in ABI Prism 377 DNA Sequencer (Applied Biosciences, NY, USA).

\subsection{Sequence Alignment and Phylogenetic Analysis}

Lagenaria siceraria sequences in FASTA format were aligned and used in the analysis of protein content of the twenty three species, the process of sequence analysis was advanced through pair wise alignment, construction of a distance matrix using Mega X. The sequences were compared to the NCBI database using Nucleotide Basic Local Alignment Search Tool (BLASTN). The results of distance matrix were sent to Excel 2007, which was finally analyzed using PAST 3.14 software for the construction of phylogenetic tree showing similarity and distance relationship between the twenty-three species. The chloroplast sequences of $L$. siceraria from NCBI data base were analysed using CHLOROBOX.

\section{Result}

Molecular result of twenty-four samples of the landraces of L. siceraria is obtained below. The result of gel electrophoresis (Figure 2) shows the quality of genomic DNA after extraction. The bands is observed in A African Bottle Gourd, B Kettle Gourd, C Caveman Club Gourd, D Base Ball Gourd, F Bushel Gourd, G Bird House Gourd, H Water Jug Gourd, I Cup Gourd, K Warted Bushel Gourd, L Extra Large Pawpaw Gourd, M Long Siphon Gourd, N Indian Gourd, O Chinese Bottle Gourd, P Mini Dipper Gourd, Q Goose Neck Gourd, S Swan Gourd, T Palm Wine Gourd, U Long Handle Dipper Gourd, V Powder Horn Gourd, W Snake Gourd and X Microphone Gourd, showing the presence of the gDNA while in E Pot Gourd, J Pennis Shield Gourd and R Nigeria Rattle Gourd showed no band. 
The polymerase chain reaction product using $r b c l$ primer (Figure 3(a)) shows a visible band at 850 bp in A African Bottle Gourd, C Caveman Club Gourd, D Base Ball Gourd, F Bushel Gourd, I Cup Gourd, K Warted Bushel Gourd, L Extra Large Pawpaw Gourd, M Long Siphon Gourd, N Indian Gourd, O Chinese Bottle Gourd, P Mini Dipper Gourd, R Nigeria Rattle Gourd, S Swan Gourd, T Palm Wine Gourd, U Long Handle Dipper Gourd, V Powder Horn Gourd, W Snake Gourd and X Microphone Gourd while B Kettle Gourd, E Pot Gourd, G

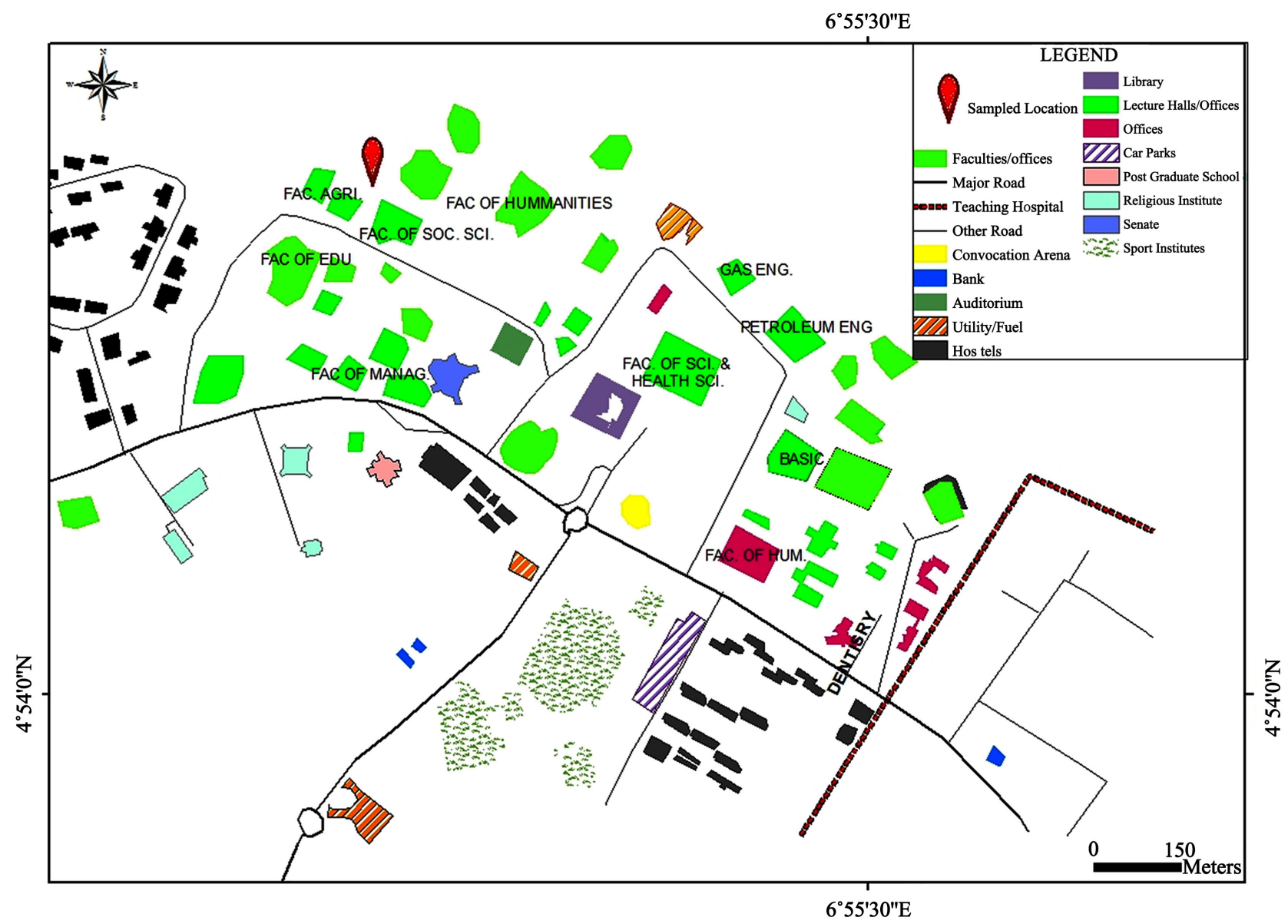

Figure 1. Map of the study area.

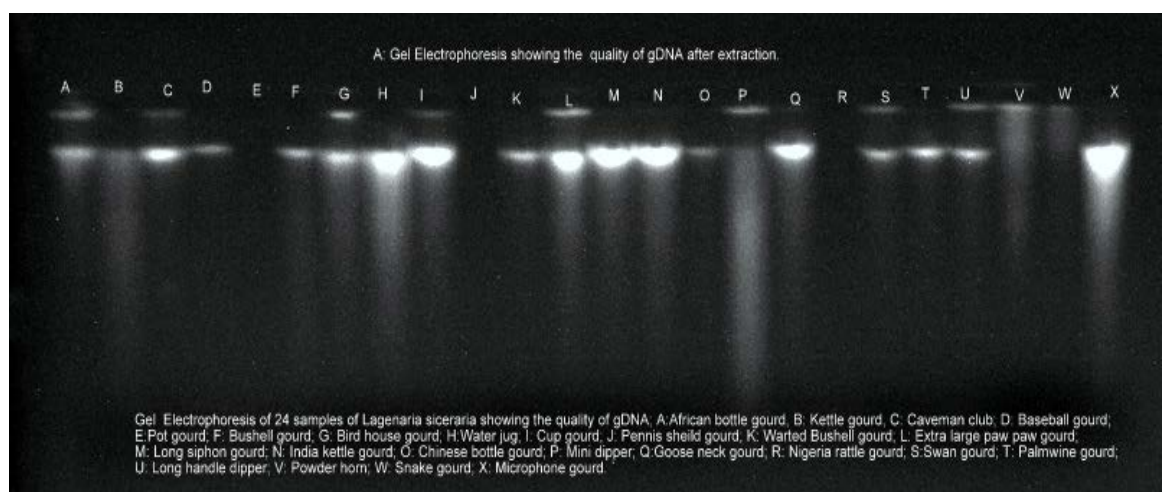

Figure 2. Gel electrophoresis: A: Showing Quality of gDNA extracted. 


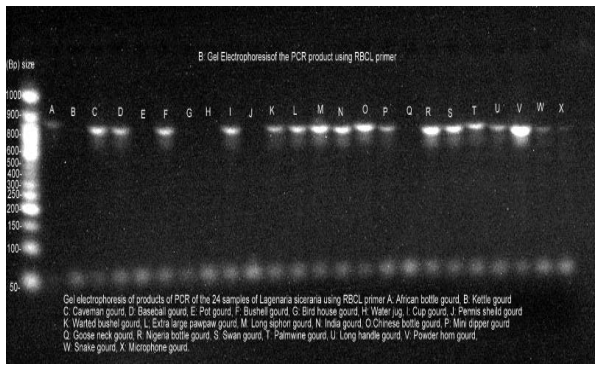

(a)

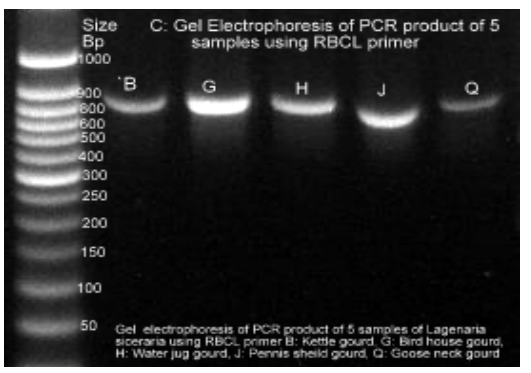

(b)

Figure 3. (a) and (b), gel electrophoresis of PCR product using rbcl primer.

Bird House Gourd, H Water Jug Gourd, J Pennis Shield Gourd and Q Goose Neck Gourd showed no band on the agarose gel electrophoresis. In the repeat of the polymerase chain reaction (Figure 3(b)) it is observed that B Kettle Gourd, G Bird House Gourd, H Water Jug Gourd, J Pennis Shield Gourd and Q Goose Neck Gourd also showed band at 850 base pair.

The quantity of DNA extracted is read using spectrophotometer as shown in Table 1 . The value ranges from 1.13 to 1.82

In Table 2 the nucleotide sequence arrangement possesses an $\mathrm{N}$ character of 720. The sequence alignment for twenty-three landraces of $L$. siceraria shows great diversity and variability in the arrangement of the bases (Table 3 ).

The result observed from BLASTN (Table 4) shows that all the sequences belong to Lagenaria siceraria with percentages ranging from $95 \%$ to $100 \%$ for query cover sequences and $98 \%$ to $100 \%$ for identity sequences with an E-value of 0.00. From the taxonomic report, sequence F-Bushel Gourd, K-Warted Bushel Gourd, U-Long Handle Dipper Gourd, I-Cup Gourd, L-Extra, M-Long Siphon Gourd, S-Swan Gourd, T-Palm Wine Gourd and W-Snake Gourd has the highest hits of 44 on Lagenaria siceraria out of 109 total value, sequence O-Chinese Bottle Gourd, P-Mini Dipper Gourd and R-Nigeria Rattle has the highest hits of 44 on L. siceraria out of the total value of 111, sequence V-Powder Horn Gourd has the highest hits of 44 on Lagenaria siceraria out of 119 total value, sequence C-Caveman Club Gourd, G-Bird House Gourd, H-Water Jug Gourd, J-Pennis Sheild, Q-Goose Neck Gourd, obtained the highest hit of 45 out of the total value 104, while sequence A-African Bottle Gourd, B Kettle Gourd, D Base Ball Gourd, has a total taxonomic value of 104 out of which 44 were identified as Lagenaria siceraria. The total taxonomic value of N-Indian Gourd, was observed as 110 with the highest hit value being 44 on Lagenaria siceraria and sequence $\mathrm{X}$-Microphone Gourd finally has a highest value hits of 44 on Lagenaria siceraria out of 105 total value.

The total nucleotide composition of the species (Table 5) varies across the different landraces. A-African Bottle Gourd is observed to have a total nucleotide composition of 689 , thymine having an average frequency of $20.1 \%$, cytosine $20.0 \%$, adenine $28.6 \%$, and guanine $22.6 \%$. The total nucleotide composition in B-Kettle Gourd is 686 with an average percentage frequency of $29.0 \%$ for thymine, $19.1 \%$ for cytosine, $28.9 \%$ for adenine and $22.4 \%$ for guanine. C-Caveman 
Table 1. Nanodrop of Lageneria siceraria (Mol.) Standl.

\begin{tabular}{|c|c|c|c|}
\hline Sample Identity & Nucleic Acid & Unit & $260 / 280$ \\
\hline A-African Bottle Gourd & 58.3 & $\mathrm{ng} / \mu \mathrm{l}$ & 1.3 \\
\hline B-Kettle Gourd & 54.7 & $\mathrm{ng} / \mu \mathrm{l}$ & 1.34 \\
\hline C-Caveman Club Gourd & 57.2 & $\mathrm{ng} / \mu \mathrm{l}$ & 1.29 \\
\hline D-Base Ball Gourd & 19.6 & $\mathrm{ng} / \mu \mathrm{l}$ & 1.82 \\
\hline E-Pot Gourd & 30.6 & $\mathrm{ng} / \mu \mathrm{l}$ & 1.23 \\
\hline F-Bushel Gourd & 33.3 & $\mathrm{ng} / \mu \mathrm{l}$ & 1.13 \\
\hline G-Bird House Gourd & 94.8 & $\mathrm{ng} / \mu \mathrm{l}$ & 1.36 \\
\hline H-Water Jug Gourd & 78.1 & $\mathrm{ng} / \mu \mathrm{l}$ & 1.47 \\
\hline I-Cup Gourd & 47.2 & $\mathrm{ng} / \mu \mathrm{l}$ & 1.23 \\
\hline J-Pennis Shield Gourd & 86.7 & $\mathrm{ng} / \mu \mathrm{l}$ & 1.46 \\
\hline K-Warted Bushel Gourd & 58.2 & $\mathrm{ng} / \mu \mathrm{l}$ & 1.31 \\
\hline L-Extra Large Pawpaw Gourd & 41.3 & $\mathrm{ng} / \mu \mathrm{l}$ & 1.16 \\
\hline M-Long Siphon Gourd & 63 & $\mathrm{ng} / \mu \mathrm{l}$ & 1.4 \\
\hline N-Indian Gourd & 70.7 & $\mathrm{ng} / \mu \mathrm{l}$ & 1.36 \\
\hline O-Chinese Bottle Gourd & 53 & $\mathrm{ng} / \mu \mathrm{l}$ & 1.48 \\
\hline P-Mini Dipper Gourd & 30.5 & $\mathrm{ng} / \mu \mathrm{l}$ & 1.2 \\
\hline Q-Goose Neck Gourd, & 78.6 & $\mathrm{ng} / \mu \mathrm{l}$ & 1.46 \\
\hline R-Nigeria Rattle Gourd & 61.2 & $\mathrm{ng} / \mu \mathrm{l}$ & 1.36 \\
\hline S-Swan Gourd & 60.5 & $\mathrm{ng} / \mu \mathrm{l}$ & 1.39 \\
\hline T-Palm Wine Gourd & 35.4 & $\mathrm{ng} / \mu \mathrm{l}$ & 1.2 \\
\hline U-Long Handle Dipper Gourd & 35 & $\mathrm{ng} / \mu \mathrm{l}$ & 1.25 \\
\hline V-Powder Horn Gourd & 116.3 & $\mathrm{ng} / \mu \mathrm{l}$ & 1.57 \\
\hline W-Snake Gourd & 65.9 & $\mathrm{ng} / \mu \mathrm{l}$ & 1.46 \\
\hline X Microphone Gourd & 98.8 & $\mathrm{ng} / \mu \mathrm{l}$ & 1.44 \\
\hline
\end{tabular}

Table 2. Nucleotide sequence arrangement.

A-African Bottle Gourd,

TTAATTAAAATTAAGCCCTCCGCGACAAAAGAGGACAAAGGTCTCTGCCATCTTGGCAGC ATTCCGAGTAACTCCTCAACCGGGAGTTCCACCTGAGGAAGCAGGGGCCGCTGTAGCTGC TGAATCTTCTACTGGTACATGGACAACTGTGTGGACCGATGGGCTTACCAGTCTTGATCG TTACAAAGGACGATGCTATGGAATCGAGCCTGTTCCTGGAGAAGAAAATCAATATATTGC TTATGTAGCTTATCCCCTAGACCTTTTTGAAGAAGGTTCTGTTACTAACATGTTTACTTC CATTGTGGGTAATGTATTTGGATTCAAGGCTCTACGTGCTCTACGTCTGGAGGATTTGCG AATCCCTACTGCTTATATTAAAACTTTCCAAGGCCCGCCTCATGGTATCCAGGTTGAAAG AGATAAATTGAACAAGTATGGTCGCCCTCTATTGGGATGTACTATTAAACCAAAATTGGG ATTATCCGCTAAGAATTATGGTAGAGCAGTTTATGAATGTTTACGCGGTGGACTTGATTT TACCAAAGATGATGAAAACGTGAATTCCCAACCATTTATGCGTTGGAGAGACCGTTTCCT ATTTTGTGCGGAAGCTATTTATAAATCACAGGCTGAAACAGGTGAAATCAAGGGACATTA CTTGAATGCTACTGCAGTACACATGCAAA,

B-Kettle Gourd,

GGCAATTTAATTAAGCCCTCCTCGAATAAGAAACCAAAGATACTGATATCTTGGCAGCAT TCCGAGTAACTCCTCAACCGGGAGTTCCACCTGAGGAAGCAGGGGCCGCTGTAGCTGCTG 
AATCTTCTACTGGTACATGGACAACTGTGTGGACCGATGGGCTTACCAGTCTTGATCGTT ACAAAGGACGATGCTATGGAATCGAGCCTGTTCCTGGAGAAGAAAATCAATATATTGCTT ATGTAGCTTATCCCCTAGACCTTTTTGAAGAAGGTTCTGTTACTAACATGTTTACTTCCA TTGTGGGTAATGTATTTGGATTCAAGGCTCTACGTGCTCTACGTCTGGAGGATTTGCGAA TCCCTACTGCTTATATTAAAACTTTCCAAGGCCCGCCTCATGGTATCCAGGTTGAAAGAG ATAAATTGAACAAGTATGGTCGCCCTCTATTGGGATGTACTATTAAACCAAAATTGGGAT TATCCGCTAAGAATTATGGTAGAGCAGTTTATGAATGTTTACGCGGTGGACTTGATTTTA CCAAAGATGATGAAAACGTGAATTCCCAACCATTTATGCGTTGGAGAGACCGTTTCCTAT TTTGTGCGGAAGCTATTTATAAATCACAGGCTGAAACAGGTGAAATCAAGGGACATTACT TGAATGCTACTGCAGTAACATGCAAA,

C-Caveman Club Gourd, AAAAATTTAGCCTTTCCCAGCGCAATATAGAGGAAACGGTCTCTGATATCTTGGCAGCAT TCCGAGTAACTCCTCAACCGGGAGTTCCACCTGAGGAAGCAGGGGCCGCTGTAGCTGCTG AATCTTCTACTGGTACATGGACAACTGTGTGGACCGATGGGCTTACCAGTCTTGATCGTT ACAAAGGACGATGCTATGGAATCGAGCCTGTTCCTGGAGAAGAAAATCAATATATTGCTT ATGTAGCTTATCCCCTAGACCTTTTTGAAGAAGGTTCTGTTACTAACATGTTTACTTCCA TTGTGGGTAATGTATTTGGATTCAAGGCTCTACGTGCTCTACGTCTGGAGGATTTGCGAA TCCCTACTGCTTATATTAAAACTTTCCAAGGCCCGCCTCATGGTATCCAGGTTGAAAGAG ATAAATTGAACAAGTATGGTCGCCCTCTATTGGGATGTACTATTAAACCAAAATTGGGAT TATCCGCTAAGAATTATGGTAGAGCAGTTTATGAATGTTTACGCGGTGGACTTGATTTTA CCAAAGATGATGAAAACGTGAATTCCCAACCATTTATGCGTTGGAGAGACCGTTTCCTAT TTTGTGCGGAAGCTATTTATAAATCACAGGCTGAAACAGGTGAAATCAAGGGACATTACT TGAATGCTACTGCAG

D-Base Ball Gourd,

AAATTTTTAATTTAGGCCTCCTCGAATATAGAAACCAAAGATACTGATATCTTGGCAGCA TTCCGAGTAACTCCTCAACCGGGAGTTCCACCTGAGGAAGCAGGGGCCGCTGTAGCTGCT GAATCTTCTACTGGTACATGGACAACTGTGTGGACCGATGGGCTTACCAGTCTTGATCGT TACAAAGGACGATGCTATGGAATCGAGCCTGTTCCTGGAGAAGAAAATCAATATATTGCT TATGTAGCTTATCCCCTAGACCTTTTTGAAGAAGGTTCTGTTACTAACATGTTTACTTCC ATTGTGGGTAATGTATTTGGATTCAAGGCTCTACGTGCTCTACGTCTGGAGGATTTGCGA ATCCCTACTGCTTATATTAAAACTTTCCAAGGCCCGCCTCATGGTATCCAGGTTGAAAGA GATAAATTGAACAAGTATGGTCGCCCTCTATTGGGATGTACTATTAAACCAAAATTGGGA TTATCCGCTAAGAATTATGGTAGAGCAGTTTATGAATGTTTACGCGGTGGACTTGATTTT ACCAAAGATGATGAAAACGTGAATTCCCAACCATTTATGCGTTGGAGAGACCGTTTCCTA TTTTGTGCGGAAGCTATTTATAAATCACAGGCTGAAACAGGTGAAATCAAGGGACATTAC TTGAATGCTACTGCAGTTAC

E-Bushel Gourd,

GTCAACTATTTATTGATTGTGTTAAGATTATAAATTGACTTATTATACTCCTGAATATGA AACCAAAGATACTGATATCTTGGCAGCATTCCGAGTAACTCCTCAACCGGGAGTTCCACC TGAGGAAGCAGGGGCCGCTGTAGCTGCTGAATCTTCTACTGGTACATGGACAACTGTGTG GACCGATGGGCTTACCAGTCTTGATCGTTACAAAGGACGATGCTATGGAATCGAGCCTGT TCCTGGAGAAGAAAATCAATATATTGCTTATGTAGCTTATCCCCTAGACCTTTTTGAAGA AGGTTCTGTTACTAACATGTTTACTTCCATTGTGGGTAATGTATTTGGATTCAAGGCTCT ACGTGCTCTACGTCTGGAGGATTTGCGAATCCCTACTGCTTATATTAAAACTTTCCAAGG CCCGCCTCATGGTATCCAGGTTGAAAGAGATAAATTGAACAAGTATGGTCGCCCTCTATT GGGATGTACTATTAAACCAAAATTGGGATTATCCGCTAAGAATTATGGTAGAGCAGTTTA TGAATGTTTACGCGGTGGACTTGATTTTACCAAAGATGATGAAAACGTGAATTCCCAACC ATTTATGCGTTGGAGAGACCGTTTCCTATTTTGTGCGGAAGCTATTTATAAATCACAGGC TGAAACAGGTGAAATCAAGGGACATTACTTGAATGCTACTGCAGGTACCATGCGAA F-Bird House Gourd, TAAAGGGATCACCTCGATTATCCTTAGGCAGCATTCCGAGTAACTCCTCAACCGGGAGTT CCACCTGAGGAAGCAGGGGCCGCTGTAGCTGCTGAATCTTCTACTGGTACATGGACAACT GTGTGGACCGATGGGCTTACCAGTCTTGATCGTTACAAAGGACGATGCTATGGAATCGAG CCTGTTCCTGGAGAAGAAAATCAATATATTGCTTATGTAGCTTATCCCCTAGACCTTTTT GAAGAAGGTTCTGTTACTAACATGTTTACTTCCATTGTGGGTAATGTATTTGGATTCAAG GCTCTACGTGCTCTACGTCTGGAGGATTTGCGAATCCCTACTGCTTATATTAAAACTTTC CAAGGCCCGCCTCATGGTATCCAGGTTGAAAGAGATAAATTGAACAAGTATGGTCGCCCT CTATTGGGATGTACTATTAAACCAAAATTGGGATTATCCGCTAAGAATTATGGTAGAGCA GTTTATGAATGTTTACGCGGTGGACTTGATTTTACCAAAGATGATGAAAACGTGAATTCC 
CAACCATTTATGCGTTGGAGAGACCGTTTCCTATTTTGTGCGGGAAGCTATTTATAAATC ACAGGCTGAAACAGGTGAAATCAAGGGACATTACTTGAATGCTACTGCAGGTACATGCAA A G-Water Jug Gourd, CAATTTCCCAGGAGTAAACTCCTCAACCGAGGAGTTCCACCTGAGGAAGCAGGGGCCGCT GTAGCTGCTGAATCTTCTACTGGTACATGGACAACTGTGTGGACCGATGGGCTTACCAGT CTTGATCGTTACAAAGGACGATGCTATGGAATCGAGCCTGTTCCTGGAGAAGAAAATCAA TATATTGCTTATGTAGCTTATCCCCTAGACCTTTTTGAAGAAGGTTCTGTTACTAACATG TTTACTTCCATTGTGGGTAATGTATTTGGATTCAAGGCTCTACGTGCTCTACGTCTGGAG GATTTGCGAATCCCTACTGCTTATATTAAAACTTTCCAAGGCCCGCCTCATGGTATCCAG GTTGAAAGAGATAAATTGAACAAGTATGGTCGCCCTCTATTGGGATGTACTATTAAACCA AAATTGGGATTATCCGCTAAGAATTATGGTAGAGCAGTTTATGAATGTTTACGCGGTGGA CTTGATTTTACCAAAAGATGATGAAAAACGTGAATTCCCAACCATTTTATGCGTTGGAGA GACCGTTTCCTATTTTTGTGCGGAAAGCTATTTATAAATCACAGGGTGAAACAGGGGAAA TCAAGGGAACATTTACTTGAATGCTACTGCAGGTACATGCAAAA H-Cup Gourd, GCACTACAGTAATAAATTGTGTTAAGATTATAAATTGACTTATTATACTCCTGAATATGA AACCAAAGATACTGATATCTTGGCAGCATTCCGAGTAACTCCTCAACCGGGAGTTCCACC TGAGGAAGCAGGGGCCGCTGTAGCTGCTGAATCTTCTACTGGTACATGGACAACTGTGTG GACCGATGGGCTTACCAGTCTTGATCGTTACAAAGGACGATGCTATGGAATCGAGCCTGT TCCTGGAGAAGAAAATCAATATATTGCTTATGTAGCTTATCCCCTAGACCTTTTTGAAGA AGGTTCTGTTACTAACATGTTTACTTCCATTGTGGGTAATGTATTTGGATTCAAGGCTCT ACGTGCTCTACGTCTGGAGGATTTGCGAATCCCTACTGCTTATATTAAAACTTTCCAAGG CCCGCCTCATGGTATCCAGGTTGAAAGAGATAAATTGAACAAGTATGGTCGCCCTCTATT GGGATGTACTATTAAACCAAAATTGGGATTATCCGCTAAGAATTATGGTAGAGCAGTTTA TGAATGTTTACGCGGTGGACTTGATTTTACCAAAGATGATGAAAACGTGAATTCCCAACC ATTTATGCGTTGGAGAGACCGTTTCCTATTTTGTGCGGAAGCTATTTATAAATCACAGGC TGAAACAGGTGAAATCAAGGGACATTACTTGAATGCTACTGCAGGTCCTTGCGA I-Pennis Shield Gourd, ACAAGGGGATTCACCCGATAATCCTTTGGCAGCATTCCGAGTAACTCCTCAACCGGGAGT TCCACCTGAGGAAGCAGGGGCCGCTGTAGCTGCTGAATCTTCTACTGGTACATGGACAAC TGTGTGGACCGATGGGCTTACCAGTCTTGATCGTTACAAAGGACGATGCTATGGAATCGA GCCTGTTCCTGGAGAAGAAAATCAATATATTGCTTATGTAGCTTATCCCCTAGACCTTTT TGAAGAAGGTTCTGTTACTAACATGTTTACTTCCATTGTGGGTAATGTATTTGGATTCAA GGCTCTACGTGCTCTACGTCTGGAGGATTTGCGAATCCCTACTGCTTATATTAAAACTTT CCAAGGCCCGCCTCATGGTATCCAGGTTGAAAGAGATAAATTGAACAAGTATGGTCGCCC TCTATTGGGATGTACTATTAAACCAAAATTGGGATTATCCGCTAAGAATTATGGTAGAGC AGTTTATGAATGTTTACGCGGTGGACTTGATTTTACCAAAGATGATGAAAACGTGAATTC CCAACCATTTATGCGTTGGAGAGACCGTTTCCTATTTTGTGCGGAAGCTATTTATAAATC ACAGGCTGAAACAGGTGAAATCAAGGGACATTACTTGAATGCTACTGCAGGTACATGCAA AAGAG

J-Warted Bushel Gourd, GCCTTCCATGTAAAAATTTTTGTTAAGGATTATAATTGACTTATTATACTCCTGAATATG AAACCAAAGATACTGATATCTTGGCAGCATTCCGAGTAACTCCTCAACCGGGAGTTCCAC CTGAGGAAGCAGGGGCCGCTGTAGCTGCTGAATCTTCTACTGGTACATGGACAACTGTGT GGACCGATGGGCTTACCAGTCTTGATCGTTACAAAGGACGATGCTATGGAATCGAGCCTG TTCCTGGAGAAGAAAATCAATATATTGCTTATGTAGCTTATCCCCTAGACCTTTTTGAAG AAGGTTCTGTTACTAACATGTTTACTTCCATTGTGGGTAATGTATTTGGATTCAAGGCTC TACGTGCTCTACGTCTGGAGGATTTGCGAATCCCTACTGCTTATATTAAAACTTTCCAAG GCCCGCCTCATGGTATCCAGGTTGAAAGAGATAAATTGAACAAGTATGGTCGCCCTCTAT TGGGATGTACTATTAAACCAAAATTGGGATTATCCGCTAAGAATTATGGTAGAGCAGTTT ATGAATGTTTACGCGGTGGACTTGATTTTACCAAAGATGATGAAAACGTGAATTCCCAAC CATTTATGCGTTGGAGAGACCGTTTCCTATTTTGTGCGGAAGCTATTTATAAATCACAGG CTGAAACAGGTGAAATCAAGGGACATTACTTGAATGCTACTGCAGGAA K-Extra Large Pawpaw Gourd, TTCTGATCAAAGTAATGATGGTTGTTAAGATTATAATTGACTTATTATACTCCTGAATAT GAAACCAAAGATACTGATATCTTGGCAGCATTCCGAGTAACTCCTCAACCGGGAGTTCCA CCTGAGGAAGCAGGGGCCGCTGTAGCTGCTGAATCTTCTACTGGTACATGGACAACTGTG TGGACCGATGGGCTTACCAGTCTTGATCGTTACAAAGGACGATGCTATGGAATCGAGCCT 
GTTCCTGGAGAAGAAAATCAATATATTGCTTATGTAGCTTATCCCCTAGACCTTTTTGAA GAAGGTTCTGTTACTAACATGTTTACTTCCATTGTGGGTAATGTATTTGGATTCAAGGCT CTACGTGCTCTACGTCTGGAGGATTTGCGAATCCCTACTGCTTATATTAAAACTTTCCAA GGCCCGCCTCATGGTATCCAGGTTGAAAGAGATAAATTGAACAAGTATGGTCGCCCTCTA TTGGGATGTACTATTAAACCAAAATTGGGATTATCCGCTAAGAATTATGGTAGAGCAGTT TATGAATGTTTACGCGGTGGACTTGATTTTACCAAAGATGATGAAAACGTGAATTCCCAA CCATTTATGCGTTGGAGAGACCGTTTCCTATTTTGTGCGGAAGCTATTTATAAATCACAG GCTGAAACAGGTGAAATCAAGGGACATTACTTGAATGCTACTGCAGTACCATGGCGAAA L-Long Siphon Gourd, GTTCAACCAATGTTATGCGGTGTGTTAAGATTATAAATTGACTTATTATACTCCTGAATA TGAAACCAAAGATACTGATATCTTGGCAGCATTCCGAGTAACTCCTCAACCGGGAGTTCC ACCTGAGGAAGCAGGGGCCGCTGTAGCTGCTGAATCTTCTACTGGTACATGGACAACTGT GTGGACCGATGGGCTTACCAGTCTTGATCGTTACAAAGGACGATGCTATGGAATCGAGCC TGTTCCTGGAGAAGAAAATCAATATATTGCTTATGTAGCTTATCCCCTAGACCTTTTTGA AGAAGGTTCTGTTACTAACATGTTTACTTCCATTGTGGGTAATGTATTTGGATTCAAGGC TCTACGTGCTCTACGTCTGGAGGATTTGCGAATCCCTACTGCTTATATTAAAACTTTCCA AGGCCCGCCTCATGGTATCCAGGTTGAAAGAGATAAATTGAACAAGTATGGTCGCCCTCT ATTGGGATGTACTATTAAACCAAAATTGGGATTATCCGCTAAGAATTATGGTAGAGCAGT TTATGAATGTTTACGCGGTGGACTTGATTTTACCAAAGATGATGAAAACGTGAATTCCCA ACCATTTATGCGTTGGAGAGACCGTTTCCTATTTTGTGCGGAAGCTATTTATAAATCACA GGCTGAAACAGGTGAAATCAAGGGACATTACTTGAATGCTACTGCAGTACCATGCGAA M-Indian Gourd, ATAGGGGGGGCCATTGCGGAACCCTGTAGCATTATAAATTGACTTATTATACTCCTGAAT ATGAAACCAAAGATACTGATATCTTGGCAGCATTCCGAGTAACTCCTCAACCGGGAGTTC CACCTGAGGAAGCAGGGGCCGCTGTAGCTGCTGAATCTTCTACTGGTACATGGACAACTG TGTGGACCGATGGGCTTACCAGTCTTGATCGTTACAAAGGACGATGCTATGGAATCGAGC CTGTTCCTGGAGAAGAAAATCAATATATTGCTTATGTAGCTTATCCCCTAGACCTTTTTG AAGAAGGTTCTGTTACTAACATGTTTACTTCCATTGTGGGTAATGTATTTGGATTCAAGG CTCTACGTGCTCTACGTCTGGAGGATTTGCGAATCCCTACTGCTTATATTAAAACTTTCC AAGGCCCGCCTCATGGTATCCAGGTTGAAAGAGATAAATTGAACAAGTATGGTCGCCCTC TATTGGGATGTACTATTAAACCAAAATTGGGATTATCCGCTAAGAATTATGGTAGAGCAG TTTATGAATGTTTACGCGGTGGACTTGATTTTACCAAAGATGATGAAAACGTGAATTCCC AACCATTTATGCGTTGGAGAGACCGTTTCCTATTTTGTGCGGAAGCTATTTATAAATCAC AGGCTGAAACAGGTGAAATCAAGGGACATTACTTGAATGCTACTGCAGTACCATGCGAAA

N-Chinese Bottle Gourd, GCCCTGCGTTGACTTATTATACTCCTGAATATGAAACCAAAGATACTGATATCTTGGCAG CATTCCGAGTAACTCCTCAACCGGGAGTTCCACCTGAGGAAGCAGGGGCCGCTGTAGCTG CTGAATCTTCTACTGGTACATGGACAACTGTGTGGACCGATGGGCTTACCAGTCTTGATC GTTACAAAGGACGATGCTATGGAATCGAGCCTGTTCCTGGAGAAGAAAATCAATATATTG CTTATGTAGCTTATCCCCTAGACCTTTTTGAAGAAGGTTCTGTTACTAACATGTTTACTT CCATTGTGGGTAATGTATTTGGATTCAAGGCTCTACGTGCTCTACGTCTGGAGGATTTGC GAATCCCTACTGCTTATATTAAAACTTTCCAAGGCCCGCCTCATGGTATCCAGGTTGAAA GAGATAAATTGAACAAGTATGGTCGCCCTCTATTGGGATGTACTATTAAACCAAAATTGG GATTATCCGCTAAGAATTATGGTAGAGCAGTTTATGAATGTTTACGCGGTGGACTTGATT TTACCAAAGATGATGAAAACGTGAATTCCCAACCATTTATGCGTTGGAGAGACCGTTTCC TATTTTGTGCGGAAGCTATTTATAAATCACAGGCTGAAACAGGTGAAATCAAGGGACATT ACTTGAATGCTACTGAG

O-Mini Dipper Gourd, ACTTTTCCCCTTCGAAATATAGAAACCAAAGATACTGATATCTTGGCAGCATTCCGAGTA ACTCCTCAACCGGGAGTTCCACCTGAGGAAGCAGGGGCCGCTGTAGCTGCTGAATCTTCT ACTGGTACATGGACAACTGTGTGGACCGATGGGCTTACCAGTCTTGATCGTTACAAAGGA CGATGCTATGGAATCGAGCCTGTTCCTGGAGAAGAAAATCAATATATTGCTTATGTAGCT TATCCCCTAGACCTTTTTGAAGAAGGTTCTGTTACTAACATGTTTACTTCCATTGTGGGT AATGTATTTGGATTCAAGGCTCTACGTGCTCTACGTCTGGAGGATTTGCGAATCCCTACT GCTTATATTAAAACTTTCCAAGGCCCGCCTCATGGTATCCAGGTTGAAAGAGATAAATTG AACAAGTATGGTCGCCCTCTATTGGGATGTACTATTAAACCAAAATTGGGATTATCCGCT AAGAATTATGGTAGAGCAGTTTATGAATGTTTACGCGGTGGACTTGATTTTACCAAAGAT 
GATGAAAACGTGAATTCCCAACCATTTATGCGTTGGAGAGACCGTTTCCTATTTTGTGCG GAAGCTATTTATAAATCACAGGCTGAAACAGGTGAAATCAAGGGACATTACTTGAATGCT ACTGAGTGCCCTTGCGAAA

P-Goose Neck Gourd,

GTCGCCTAATCCGTTTGGCAGCATTCCGAGTAACTCCTCAACCGGGAGTTCCACCTGAGG AAGCAGGGGCCGCTGTAGCTGCTGAATCTTCTACTGGTACATGGACAACTGTGTGGACCG ATGGGCTTACCAGTCTTGATCGTTACAAAGGACGATGCTATGGAATCGAGCCTGTTCCTG GAGAAGAAAATCAATATATTGCTTATGTAGCTTATCCCCTAGACCTTTTTGAAGAAGGTT CTGTTACTAACATGTTTACTTCCATTGTGGGTAATGTATTTGGATTCAAGGCTCTACGTG CTCTACGTCTGGAGGATTTGCGAATCCCTACTGCTTATATTAAAACTTTCCAAGGCCCGC CTCATGGTATCCAGGTTGAAAGAGATAAATTGAACAAGTATGGTCGCCCTCTATTGGGAT GTACTATTAAACCAAAATTGGGATTATCCGCTAAGAATTATGGTAGAGCAGTTTATGAAT GTTTACGCGGTGGACTTGATTTTACCAAAGATGATGAAAACGTGAATTCCCAACCATTTA TGCGTTGGAGAGACCGTTTCCTATTTTGTGCGGAAGCTATTTATAAATCACAGGCTGAAA CAGGTGAAATCAAGGGACATTACTTGAATGCTACTGCAGGTTACATGCGAA

Q-Nigeria Rattle Gourd, AAGCGAGGGCCCTTTGCCTAACCTGTTTAGCCTGTGATGTATAATTATCTTCCTGAATAT GAAACCAAAGATACTGATATCTTGGCAGCATTCCGAGTAACTCCTCAACCGGGAGTTCCA CCTGAGGAAGCAGGGGCCGCTGTAGCTGCTGAATCTTCTACTGGTACATGGACAACTGTG TGGACCGATGGGCTTACCAGTCTTGATCGTTACAAAGGACGATGCTATGGAATCGAGCCT GTTCCTGGAGAAGAAAATCAATATATTGCTTATGTAGCTTATCCCCTAGACCTTTTTGAA GAAGGTTCTGTTACTAACATGTTTACTTCCATTGTGGGTAATGTATTTGGATTCAAGGCT CTACGTGCTCTACGTCTGGAGGATTTGCGAATCCCTACTGCTTATATTAAAACTTTCCAA GGCCCGCCTCATGGTATCCAGGTTGAAAGAGATAAATTGAACAAGTATGGTCGCCCTCTA TTGGGATGTACTATTAAACCAAAATTGGGATTATCCGCTAAGAATTATGGTAGAGCAGTT TATGAATGTTTACGCGGTGGACTTGATTTTACCAAAGATGATGAAAACGTGAATTCCCAA CCATTTATGCGTTGGAGAGACCGTTTCCTATTTTGTGCGGAAGCTATTTATAAATCACAG GCTGAAACAGGTGAAATCAAGGGACATTACTTGAATGCTACTGAG

S-Swan Gourd, GCCATCCGGCTTAAGGAAGTCCGTTAAAGATTATAAATTGACTTATTATACTCCTGAATA TGAAACCAAAGATACTGATATCTTGGCAGCATTCCGAGTAACTCCTCAACCGGGAGTTCC ACCTGAGGAAGCAGGGGCCGCTGTAGCTGCTGAATCTTCTACTGGTACATGGACAACTGT GTGGACCGATGGGCTTACCAGTCTTGATCGTTACAAAGGACGATGCTATGGAATCGAGCC TGTTCCTGGAGAAGAAAATCAATATATTGCTTATGTAGCTTATCCCCTAGACCTTTTTGA AGAAGGTTCTGTTACTAACATGTTTACTTCCATTGTGGGTAATGTATTTGGATTCAAGGC TCTACGTGCTCTACGTCTGGAGGATTTGCGAATCCCTACTGCTTATATTAAAACTTTCCA AGGCCCGCCTCATGGTATCCAGGTTGAAAGAGATAAATTGAACAAGTATGGTCGCCCTCT ATTGGGATGTACTATTAAACCAAAATTGGGATTATCCGCTAAGAATTATGGTAGAGCAGT TTATGAATGTTTACGCGGTGGACTTGATTTTACCAAAGATGATGAAAACGTGAATTCCCA ACCATTTATGCGTTGGAGAGACCGTTTCCTATTTTGTGCGGAAGCTATTTATAAATCACA GGCTGAAACAGGTGAAATCAAGGGACATTACTTGAATGCTACTGCAGTACCATGCGAA T-Palm Wine Gourd, TTTCGGGTTAATACTTGTGTTAAGATTATAAATTGACTTATTATACTCCTGAATATGAAA CCAAAGATACTGATATCTTGGCAGCATTCCGAGTAACTCCTCAACCGGGAGTTCCACCTG AGGAAGCAGGGGCCGCTGTAGCTGCTGAATCTTCTACTGGTACATGGACAACTGTGTGGA CCGATGGGCTTACCAGTCTTGATCGTTACAAAGGACGATGCTATGGAATCGAGCCTGTTC CTGGAGAAGAAAATCAATATATTGCTTATGTAGCTTATCCCCTAGACCTTTTTGAAGAAG GTTCTGTTACTAACATGTTTACTTCCATTGTGGGTAATGTATTTGGATTCAAGGCTCTAC GTGCTCTACGTCTGGAGGATTTGCGAATCCCTACTGCTTATATTAAAACTTTCCAAGGCC CGCCTCATGGTATCCAGGTTGAAAGAGATAAATTGAACAAGTATGGTCGCCCTCTATTGG GATGTACTATTAAACCAAAATTGGGATTATCCGCTAAGAATTATGGTAGAGCAGTTTATG AATGTTTACGCGGTGGACTTGATTTTACCAAAGATGATGAAAACGTGAATTCCCAACCAT TTATGCGTTGGAGAGACCGTTTCCTATTTTGTGCGGAAGCTATTTATAAATCACAGGCTG AAACAGGTGAAATCAAGGGACATTACTTGAATGCTACTGCAGTACCCTTGCGAA 
U-Long Handle Dipper Gourd, GCCTTCAATGGTAAAATTGTGTTAAGATTATAAATTGACTTATTATACTCCTGAATATGA AACCAAAGATACTGATATCTTGGCAGCATTCCGAGTAACTCCTCAACCGGGAGTTCCACC TGAGGAAGCAGGGGCCGCTGTAGCTGCTGAATCTTCTACTGGTACATGGACAACTGTGTG GACCGATGGGCTTACCAGTCTTGATCGTTACAAAGGACGATGCTATGGAATCGAGCCTGT TCCTGGAGAAGAAAATCAATATATTGCTTATGTAGCTTATCCCCTAGACCTTTTTGAAGA AGGTTCTGTTACTAACATGTTTACTTCCATTGTGGGTAATGTATTTGGATTCAAGGCTCT ACGTGCTCTACGTCTGGAGGATTTGCGAATCCCTACTGCTTATATTAAAACTTTCCAAGG CCCGCCTCATGGTATCCAGGTTGAAAGAGATAAATTGAACAAGTATGGTCGCCCTCTATT GGGATGTACTATTAAACCAAAATTGGGATTATCCGCTAAGAATTATGGTAGAGCAGTTTA TGAATGTTTACGCGGTGGACTTGATTTTACCAAAGATGATGAAAACGTGAATTCCCAACC ATTTATGCGTTGGAGAGACCGTTTCCTATTTTGTGCGGAAGCTATTTATAAATCACAGGC TGAAACAGGTGAAATCAAGGGACATTACTTGAATGCTACTGCAGTAACCTTGCGAAA

V-Powder Horn Gourd, TTTGGGGGTCAATGCTGGTGTTAAGATTATAAATTGACTTATTATACTCCTGAATATGAA ACCAAAGATACTGATATCTTGGCAGCATTCCGAGTAACTCCTCAACCGGGAGTTCCACCT GAGGAAGCAGGGGCCGCTGTAGCTGCTGAATCTTCTACTGGTACATGGACAACTGTGTGG ACCGATGGGCTTACCAGTCTTGATCGTTACAAAGGACGATGCTATGGAATCGAGCCTGTT CCTGGAGAAGAAAATCAATATATTGCTTATGTAGCTTATCCCCTAGACCTTTTTGAAGAA GGTTCTGTTACTAACATGTTTACTTCCATTGTGGGTAATGTATTTGGATTCAAGGCTCTA CGTGCTCTACGTCTGGAGGATTTGCGAATCCCTACTGCTTATATTAAAACTTTCCAAGGC CCGCCTCATGGTATCCAGGTTGAAAGAGATAAATTGAACAAGTATGGTCGCCCTCTATTG GGATGTACTATTAAACCAAAATTGGGATTATCCGCTAAGAATTATGGTAGAGCAGTTTAT GAATGTTTACGCGGTGGACTTGATTTTACCAAAGATGATGAAAACGTGAATTCCCAACCA TTTATGCGTTGGAGAGACCGTTTCCTATTTTGTGCGGAAGCTATTTATAAATCACAGGCT GAAACAGGTGAAATCAAGGGACATTACTTGAATGCTACTGAG

W-Snake Gourd, CATTCATTGTCTATAATTCTGTTAAGATTATAATTGACTTATTATACTCCTGAATATGAA ACCAAAGATACTGATATCTTGGCAGCATTCCGAGTAACTCCTCAACCGGGAGTTCCACCT GAGGAAGCAGGGGCCGCTGTAGCTGCTGAATCTTCTACTGGTACATGGACAACTGTGTGG ACCGATGGGCTTACCAGTCTTGATCGTTACAAAGGACGATGCTATGGAATCGAGCCTGTT CCTGGAGAAGAAAATCAATATATTGCTTATGTAGCTTATCCCCTAGACCTTTTTGAAGAA GGTTCTGTTACTAACATGTTTACTTCCATTGTGGGTAATGTATTTGGATTCAAGGCTCTA CGTGCTCTACGTCTGGAGGATTTGCGAATCCCTACTGCTTATATTAAAACTTTCCAAGGC CCGCCTCATGGTATCCAGGTTGAAAGAGATAAATTGAACAAGTATGGTCGCCCTCTATTG GGATGTACTATTAAACCAAAATTGGGATTATCCGCTAAGAATTATGGTAGAGCAGTTTAT GAATGTTTACGCGGTGGACTTGATTTTACCAAAGATGATGAAAACGTGAATTCCCAACCA TTTATGCGTTGGAGAGACCGTTTCCTATTTTGTGCGGAAGCTATTTATAAATCACAGGCT GAAACAGGTGAAATCAAGGGACATTACTTGAATGCTACTGCAGTAAC

X Microphone Gourd, GCATCAATGTGGGAGATTTCTTTGTGGGGTATGGTTGACTTATTATACTCCTGAATATGA AACCAAAGATACTGATATCTTGGCAGCATTCCGAGTAACTCCTCAACCGGGAGTTCCACC TGAGGAAGCAGGGGCCGCTGTAGCTGCTGAATCTTCTACTGGTACATGGACAACTGTGTG GACCGATGGGCTTACCAGTCTTGATCGTTACAAAGGACGATGCTATGGAATCGAGCCTGT TCCTGGAGAAGAAAATCAATATATTGCTTATGTAGCTTATCCCCTAGACCTTTTTGAAGA AGGTTCTGTTACTAACATGTTTACTTCCATTGTGGGTAATGTATTTGGATTCAAGGCTCT ACGTGCTCTACGTCTGGAGGATTTGCGAATCCCTACTGCTTATATTAAAACTTTCCAAGG CCCGCCTCATGGTATCCAGGTTGAAAGAGATAAATTGAACAAGTATGGTCGCCCTCTATT GGGATGTACTATTAAACCAAAATTGGGATTATCCGCTAAGAATTATGGTAGAGCAGTTTA TGAATGTTTACGCGGTGGACTTGATTTTACCAAAGATGATGAAAACGTGAATTCCCAACC ATTTATGCGTTGGAGAGACCGTTTCCTATTTTGTGCGGAAGCTATTTATAAATCACAGGC TGAAACAGGTGAAATCAAAGGGACATTACTTGAATGCTACTGCAGGTACCATGCAGAAA 
Table 3. Sequence alignment for twenty-three (23) fruit shapes of L. siceraria (Mol.) Standl. Complex.

\begin{tabular}{|c|c|c|}
\hline $\mathrm{S} / \mathrm{N}$ & TAXA & DNA SEQUENCE \\
\hline 1 & A-African Bottle Gourd & TTAATTAAAATTAAGCCCTCCGCGACAAAAGAGGACAAAGGTCTCTGCCATCTTGGCAGC \\
\hline 2 & B-Kettle Gourd & GGCAATTTAATTAAGCCCTCCTCGAATAAGAAACCAAAGATACTGATATCTTGGCAGCAT \\
\hline 3 & C-Caveman Club Gourd & AAAAATTTAGCCTTTCCCAGCGCAATATAGAGGAAACGGTCTCTGATATCTTGGCAGCAT \\
\hline 4 & D-Base Ball Gourd & AAATTTTTAATTTAGGCCTCCTCGAATATAGAAACCAAAGATACTGATATCTTGGCAGCA \\
\hline 5 & F-Bushel Gourd & GTCAACTATTTATTGATTGTGTTAAGATTATAAATTGACTTATTATACTCCTGAATATGA \\
\hline 6 & G-Bird House Gourd & TAAAGGGATCACCTCGATTATCCTTAGGCAGCATTCCGAGTAACTCCTCAACCGGGAGTT \\
\hline 7 & H-Water Jug Gourd & CAATTTCCCAGGAGTAAACTCCTCAACCGAGGAGTTCCACCTGAGGAAGCAGGGGCCGCT \\
\hline 8 & I-Cup Gourd & GCACTACAGTAATAAATTGTGTTAAGATTATAAATTGACTTATTATACTCCTGAATATGA \\
\hline 9 & J-Pennis Shield Gourd & ACAAGGGGATTCACCCGATAATCCTTTGGCAGCATTCCGAGTAACTCCTCAACCGGGAGT \\
\hline 10 & K-Warted Bushel Gourd & GCCTTCCATGTAAAAATTTTTGTTAAGGATTATAATTGACTTATTATACTCCTGAATATG \\
\hline 11 & L-Extra Large Pawpaw Gourd & TTCTGATCAAAGTAATGATGGTTGTTAAGATTATAATTGACTTATTATACTCCTGAATAT \\
\hline 12 & M-Long Siphon Gourd & GTTCAACCAATGTTATGCGGTGTGTTAAGATTATAAATTGACTTATTATACTCCTGAATA \\
\hline 13 & N-Indian Gourd & ATAGGGGGGGCCATTGCGGAACCCTGTAGCATTATAAATTGACTTATTATACTCCTGAAT \\
\hline 14 & O-Chinese Bottle Gourd & GCCCTGCGTTGACTTATTATACTCCTGAATATGAAACCAAAGATACTGATATCTTGGCAG \\
\hline 15 & P-Mini Dipper Gourd & ACTTTTCCCCTTCGAAATATAGAAACCAAAGATACTGATATCTTGGCAGCATTCCGAGTA \\
\hline 16 & Q-Goose Neck Gourd, & GTCGCCTAATCCGTTTGGCAGCATTCCGAGTAACTCCTCAACCGGGAGTTCCACCTGAGG \\
\hline 17 & R-Nigeria Rattle Gourd & AAGCGAGGGCCCTTTGCCTAACCTGTTTAGCCTGTGATGTATAATTATCTTCCTGAATAT \\
\hline 18 & S-Swan Gourd & GCCATCCGGCTTAAGGAAGTCCGTTAAAGATTATAAATTGACTTATTATACTCCTGAATA \\
\hline 19 & T-Palm Wine Gourd & TTTCGGGTTAATACTTGTGTTAAGATTATAAATTGACTTATTATACTCCTGAATATGAAA \\
\hline 20 & U-Long Handle Dipper Gourd & GCCTTCAATGGTAAAATTGTGTTAAGATTATAAATTGACTTATTATACTCCTGAATATGA \\
\hline 21 & V-Powder Horn Gourd & TTTGGGGGTCAATGCTGGTGTTAAGATTATAAATTGACTTATTATACTCCTGAATATGAA \\
\hline 22 & W-Snake Gourd & CATTCATTGTCTATAATTCTGTTAAGATTATAATTGACTTATTATACTCCTGAATATGAA \\
\hline 23 & X Microphone Gourd & GCATCAATGTGGGAGATTTCTTTGTGGGGTATGGTTGACTTATTATACTCCTGAATATGA \\
\hline
\end{tabular}

Table 4. Nucleotide basic local alignment search tool (BLAST N).

\begin{tabular}{|c|c|c|c|c|c|c|c|}
\hline $\mathrm{S} / \mathrm{N}$ & NAME & FRUIT SHAPE & $\begin{array}{c}\text { TOTAL TAXONOMY } \\
\text { VALUE }\end{array}$ & $\begin{array}{l}\text { HIGHEST HIT } \\
\text { VALUE }\end{array}$ & QUERY \% & IDENTITY \% & E Value \\
\hline 1 & Awala A & African Bottle Gourd & 104 & 44 & 91 & 99 & 0 \\
\hline 2 & Awala B & Kettle Gourd & 104 & 44 & 97 & 99 & 0 \\
\hline 3 & Awala C & Caveman Club Gourd & 104 & 45 & 93 & 100 & 0 \\
\hline 4 & Awala D & Baseball Gourd & 104 & 44 & 97 & 99 & 0 \\
\hline 5 & Awala $\mathrm{F}$ & Bushell Gourd & 109 & 44 & 97 & 99 & 0 \\
\hline 6 & Awala G & Bird House Gourd & 104 & 45 & 96 & 99 & 0 \\
\hline 7 & Awala $\mathrm{H}$ & Water Jug Gourd & 104 & 45 & 97 & 98 & 0 \\
\hline 8 & Awala I & Cup Gourd & 109 & 44 & 97 & 99 & 0 \\
\hline 9 & Awala J & Pennis Shield Gourd & 104 & 45 & 95 & 100 & 0 \\
\hline
\end{tabular}




\section{Continued}

\begin{tabular}{|c|c|c|c|c|c|c|c|}
\hline 10 & Awala K & Warted Bushel Gourd & 109 & 44 & 96 & 99 & 0 \\
\hline 11 & Awala L & Extralarge Pawpaw Gourd & 109 & 44 & 97 & 99 & 0 \\
\hline 12 & Awala M & Long siphon Gourd & 109 & 44 & 97 & 99 & 0 \\
\hline 13 & Awala N & Indian Gourd & 110 & 44 & 95 & 99 & 0 \\
\hline 14 & Awala O & Chinese Bottle Gourd & 111 & 44 & 98 & 100 & 0 \\
\hline 15 & Awala P & Mini Dipper Gourd & 111 & 44 & 95 & 99 & 0 \\
\hline 16 & Awala Q & Goose Neck Gourd & 104 & 45 & 97 & 99 & 0 \\
\hline 17 & Awala R & Nigeria Rattle Gourd & 111 & 44 & 93 & 99 & 0 \\
\hline 18 & Awala S & Swan Gourd & 109 & 44 & 96 & 99 & 0 \\
\hline 19 & Awala T & Palm Wine Gourd & 109 & 44 & 96 & 99 & 0 \\
\hline 20 & Awala U & Long Handle Dipper Gourd & 109 & 44 & 95 & 99 & 0 \\
\hline 21 & Awala V & Powder Horn Gourd & 119 & 44 & 98 & 99 & 0 \\
\hline 22 & Awala W & Snake Gourd & 109 & 44 & 96 & 99 & 0 \\
\hline 23 & Awala X & Microphone Gourd & 105 & 44 & 95 & 99 & 0 \\
\hline
\end{tabular}

Table 5. Nucleotide composition frequencies of Lageneria siceraria (Mol.) Standl.

\begin{tabular}{|c|c|c|c|c|c|c|c|c|c|c|c|c|c|c|c|c|c|c|c|c|}
\hline & (U) & $\mathrm{C}$ & A & G & otal & T-1 & C-1 & A-1 & G-1 & s \# 1 & T-2 & $\mathrm{C}-2$ & A-2 & G-2 & Pos \#2 & T-3 & C-3 & A-3 & G-3 I & ss \#3 \\
\hline $1 \mathrm{~F}$ & & .0 & . & 22.6 & 689 & 40.9 & 9 & 30.4 & 8 & 230 & .4 & 20.4 & 2 & 3.9 & 0 & 24.9 & 25.8 & .1 & 19.2 & 29 \\
\hline & 29.0 & .7 & 9 & 22.4 & 86 & 4.5 & 25.3 & 1.4 & .8 & & .9 & 14.4 & .6 & 13.1 & 29 & 0.6 & 19.3 & 4.6 & 35.5 & 228 \\
\hline IA & 3 & 19.7 & 0 & .0 & 675 & 24.9 & 25.3 & 30.7 & .1 & 225 & .7 & 14.2 & 3.9 & 4.2 & 5 & 0.4 & 19.6 & 4.4 & 35.6 & 225 \\
\hline D IF & 29.9 & .4 & 3.4 & 22.4 & 680 & 21.1 & 18.1 & 25.6 & 2 & 227 & 5.1 & 26.4 & 30.4 & 18.1 & 227 & 43.4 & 13.7 & 29.2 & 13.7 & 226 \\
\hline . & 30.3 & 10. & 28.6 & 22.2 & 716 & 44.8 & 12.6 & 28.9 & 13.8 & 239 & 22.6 & 18.0 & 25.5 & 33.9 & 239 & 23.5 & 26.1 & 31.5 & 18.9 & 238 \\
\hline$t$ & 29.2 & 19.7 & 28.0 & 23.1 & 661 & 23.1 & 20.4 & 24.0 & 32.6 & 221 & 4.1 & 22.7 & 30.5 & 22.7 & 220 & 40.5 & 15. & 29.5 & 14.1 & 220 \\
\hline$N A L A \mathrm{H}$ & 29.2 & 19.1 & 28.7 & 23.0 & 644 & 22.3 & 21.4 & 27.0 & 29.3 & 215 & 26.0 & 20.0 & 29.8 & 24.2 & 215 & 39.3 & 15.9 & 29.4 & 15.4 & 214 \\
\hline WAI & 30.0 & 19.0 & 28.7 & 22.3 & 714 & 45.0 & 13.4 & 28.2 & 134 & 238 & 22.3 & 18.5 & 48 & $345>$ & 238 & 22.7 & 25 & 3.2 & 18.9 & 238 \\
\hline WALA J R & 28.9 & 19.7 & 28.1 & 23.3 & 665 & 43.2 & 13.1 & 30.2 & 13.5 & 222 & 20.3 & 19.8 & 23.9 & 36.0 & 222 & 23.1 & 26.2 & 30.3 & 20.4 & 221 \\
\hline AWALA K 1F & 30.2 & 18.9 & 2 & 22 & 70 & 23 & 25 & 31 & 19 & 23 & 44.5 & 12.7 & 30.1 & 12.7 & 236 & 22.5 & 18 & 6 & 34.3 & 236 \\
\hline $1 \mathrm{~F}$ & 30.0 & 18.8 & 28.8 & 22.4 & 719 & 24.2 & 18.3 & 24.2 & 33.3 & 240 & 24.2 & 25.4 & 30.8 & 19.6 & 240 & 41.8 & 12.6 & 31.4 & 14.2 & 239 \\
\hline AWALA M 1F & 29. & 19.1 & 28.6 & 22. & 718 & 43. & 13 & 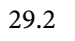 & 13.8 & 240 & 22.6 & 18.4 & 23.8 & 35.1 & 239 & 23.4 & 2 & 2.6 & 18.4 & 239 \\
\hline WALA & 29.2 & 19.4 & 28.5 & 22.9 & 720 & 22.9 & 25.4 & 32.1 & 19.6 & 240 & 43.3 & 13.8 & 28.8 & 14.2 & 240 & 21.3 & 19.2 & 24.6 & 35.0 & 240 \\
\hline & 2 & 19.6 & 27.9 & 22 & 677 & 23.5 & 29 & 31.4 & 18.6 & 226 & 44.7 & 13.3 & 27.9 & 14.2 & & 20.9 & $1 S$ & .4 & 35.6 & 225 \\
\hline WAL & 29.5 & 19.9 & 28.1 & 22.5 & 679 & 20.3 & 19.8 & 25.1 & 34.8 & 227 & 24.3 & 25.7 & 30.5 & 19.5 & 226 & 43.8 & 14.2 & 28.8 & 13.3 & 226 \\
\hline th & 29 & 19 & 27.3 & 23.3 & 651 & 24.9 & 24 & 29.0 & 21.2 & 217 & 3 & 13 & 29.0 & 13.8 & & 3 & 2 & 0 & 0 & 217 \\
\hline WA & 29.8 & 19.6 & 27.8 & 22.8 & 705 & 20.9 & 20.0 & 23.8 & 35.3 & 235 & 24.3 & 25.5 & 31.9 & 18.3 & 235 & 44.3 & 13.2 & 27.7 & 14.9 & 235 \\
\hline AWALA & 2 & 19.5 & 28.7 & 22.4 & 718 & 42 & 13 & 29.6 & 13.8 & 2 & 21.8 & 18 & 24.7 & 35.1 & 239 & 4 & 2 & .8 & 18.4 & 239 \\
\hline AWALA T 1F & 30.4 & 19.0 & 28.3 & 22.3 & 714 & 23. & 25 & 31.9 & 18.5 & 238 & 44.1 & 13.0 & 29.0 & 13.9 & 8 & 23.1 & 18.5 & 23.9 & 34.5 & 238 \\
\hline AWAL & 30.0 & 19 & 28.9 & 22.2 & 7 & 43 & 12 & 29.7 & 14.2 & 2 & 22.2 & 18.4 & 25.1 & .3 & 239 & 24.3 & 2 & 31.8 & 18.0 & 239 \\
\hline WAIA V $1 F$ & 30.2 & 18.7 & 28.2 & 22.9 & 702 & 22 & 18 & 23.9 & 35.0 & 234 & 23.1 & 24.8 & 32 & 19.7 & $232>>3$ & 44.9 & 8 & 28.2 & .1 & 234 \\
\hline AWAI & 30.6 & 19.1 & 28.6 & 21.8 & 707 & 22.5 & 18.6 & 25.0 & 33.9 & 236 & 25.4 & 26.3 & 30.5 & 17.8 & 236 & 43.8 & 12.3 & 30.2 & 13.6 & 235 \\
\hline AWALA X $1 F$ & 29.8 & 18.9 & 28.1 & 23.2 & 719 & 42 & 13 & 20 & 14.2 & $24+2-1$ & 22.5 & 19.2 & $23+>$ & 34.6 & 240 & 23.8 & 3 & 31.0 & 20.9 & 239 \\
\hline Avg. & 29.7 & 19.3 & 28.4 & 22.6 & 5.0 & 30.2 & 19.0 & 28.4 & 22.4 & 231.9 & 29.3 & 19.3 & 28.1 & 23.3 & 231.7 & 29 & 19.6 & 28.6 & 22.2 & 231.3 \\
\hline
\end{tabular}


Club Gourd have a nucleotide composition of 675 with an average percentage frequency of thymine $29.3 \%$, cytosine $19.7 \%$, adenine $28.0 \%$ and guanine $23.0 \%$. The nucleotide composition of D-Base Ball Gourd consist of 680 nucleotides with an average percentage frequency of thymine $29.9 \%$, cytosine $19.4 \%$, adenine $28.4 \%$ and guanine $22.4 \%$, in F-Bushel Gourd, the nucleotide composition is 716 while thymine $30.3 \%$, cytosine $18.9 \%$, adenine $28.6 \%$ and guanine $22.2 \%$, G-Bird House Gourd has a total composition of nucleotide as 661 , thymine $29.2 \%$, cytosine $19.7 \%$, adenine $28.0 \%$ and guanine $23.1 \%$, the total composition of nucleotide in $\mathrm{H}$-Water Jug Gourd is 664 , thymine $29.2 \%$, cytosine $19.1 \%$, adenine $28.7 \%$ and guanine $23.0 \%$, in I-Cup Gourd the total nucleotide composition is 714 , thymine $30.0 \%$, cytosine $19.0 \%$, adenine $28.7 \%$ and guanine $22.3 \%$, the total nucleotide composition is 665 in J-Pennis Shield Gourd, thymine 28.9\%, cytosine $19.7 \%$, adenine $28.1 \%$ and guanine $23.3 \%$, also in K-Warted Bushel Gourd the total nucleotide composition is 708 , thymine $30.2 \%$, cytosine $18.9 \%$, adenine $28.7 \%$ and guanine $22.2 \%$, the total nucleotide composition of L-Extra Large Pawpaw Gourd is 719 were the percentage frequency of the bases are thymine $30.0 \%$, cytosine $18.8 \%$, adenine $28.8 \%$ and guanine $22.4 \%$, M-Long Siphon Gourd has a total nucleotide composition of 718 and the percentage frequency of is thymine $29.9 \%$, cytosine $19.1 \%$, adenine $28.6 \%$ and guanine $22.4 \%$, in $\mathrm{N}$-Indian Gourd, the percentage frequency of thymine $29.2 \%$, cytosine $19.4 \%$, adenine $28.5 \%$ and guanine $22.9 \%$, it possesses a total nucleotide composition of 720. O-Chinese Bottle Gourd has a total nucleotide composition as 677 while the percentage frequency of thymine is $29.7 \%$, cytosine $19.6 \%$, adenine $27.9 \%$ and guanine $22.7 \%$, in P-Mini Dipper Gourd the total nucleotide composition equals 679 nucleotides while the percentage frequency of the nucleotides has thymine to be equal to $29.5 \%$, cytosine $19.9 \%$, adenine $28.1 \%$ and guanine $22.5 \%$, Q-Goose Neck Gourd, the total composition of nucleotide is 651 while the percentage frequency of each bases are thymine 29.5\%, cytosine $19.8 \%$, adenine $27.3 \%$ and guanine $23.3 \%$, in R-Nigeria Rattle Gourd, the total nucleotide composition is 705 and the percentage frequency of the nucleotide is thymine $29.8 \%$, cytosine $19.6 \%$, adenine $27.8 \%$ and guanine $22.8 \%$. The total nucleotide composition of S-Swan Gourd is 718 having a percentage frequency as thymine $29.4 \%$, cytosine $19.5 \%$, adenine $28.7 \%$ and guanine $22.4 \%$, T-Palm Wine Gourd the total nucleotide composition is 714 were the percentage frequency of the nucleotides are thymine $30.4 \%$, cytosine $19.0 \%$, adenine $28.3 \%$ and guanine $22.3 \%$, in U-Long Handle Dipper Gourd the frequency of the nucleotides are thymine $30.0 \%$, cytosine $19.0 \%$, adenine $28.9 \%$ and guanine $22.4 \%$, and a total nucleotide composition of 717. V-Powder Horn Gourd posseses a total nucleotide content of 702 , with a percentage frequency of thymine $30.2 \%$, cytosine $18.7 \%$, adenine $28.2 \%$ and guanine $22.9 \%$, in $\mathrm{W}$-Snake Gourd the total nucleotide composition is 707 while the percentage frequency of the bases are: thymine $30.6 \%$, cytosine $19.1 \%$, adenine $28.6 \%$ and guanine $21.8 \%$, and finally, X-Microphone Gourd is observed to have a total nucleotide composition of 719 with thymine as $29.8 \%$, cytosine $18.9 \%$, adenine $28.1 \%$ and guanine $23.2 \%$, percentage frequencies. 
Estimates of Evolutionary Divergence between Sequences are shown in the data matrix (Table 6). The number of base differences per site from between sequences is shown. Standard error estimate(s) are shown above the diagonal and were obtained by using analytical formulas. The rate variation among sites was modelled with a gamma distribution (shape parameter $=0.05$ ). The analysis involved 23 nucleotide sequences. Codon positions included were 1 st +2 nd + 3rd + Noncoding. All ambiguous positions were removed for each sequence pair. There were a total of 720 positions in the final dataset. Evolutionary analyses are conducted in MEGA X [15].

The phylogenetic tree (Figure 4) shows great diversity and similarity in Long handle dipper $-U$ and Microphone gourd-X are out group from the root, and meets at similarity scale of 0.7 , while Kettle gourd-B and Caveman club gourd-C are out group from the second root of the tree meeting at a similarity scale of 1.0. The second root has two main clusters that meets at different similarity scale. The first cluster of the second root (R2a), African bottle gourd-A is seen as out group at 0.65 while Snake gourd-W and Powder horn gourd-V clustered together at 1.0 similarity scale. Swan gourd-S and palmwine gourd-T meets at 0.89 while Mini dipper gourd-P and Goose neck gourd-Q clustered at 1.0, Nigerian gourd- $\mathrm{R}$ is observed as an out group at 0.85 similarity scale. In the second cluster of the second root (R2b), Cup gourd-I was an out group at 0.85 while Baseball gourd-D, Bird house gourd-G, Water jug gourd-H, Penis shield gourd-J, Warted bushel gourd-K, Extra-large pawpaw gourd-L, Long siphon gourd-M, Indian gourd- $\mathrm{N}$ and Chinese gourd-O met at 1.0 similarity scale.

\section{Discussion}

According to [6], the concentration of macromolecular studies was geared towards DNA and RNA hence, molecular systematics deals with the utilization of nucleic acid data. Genetic materials have been used in the understanding of the

Similarity

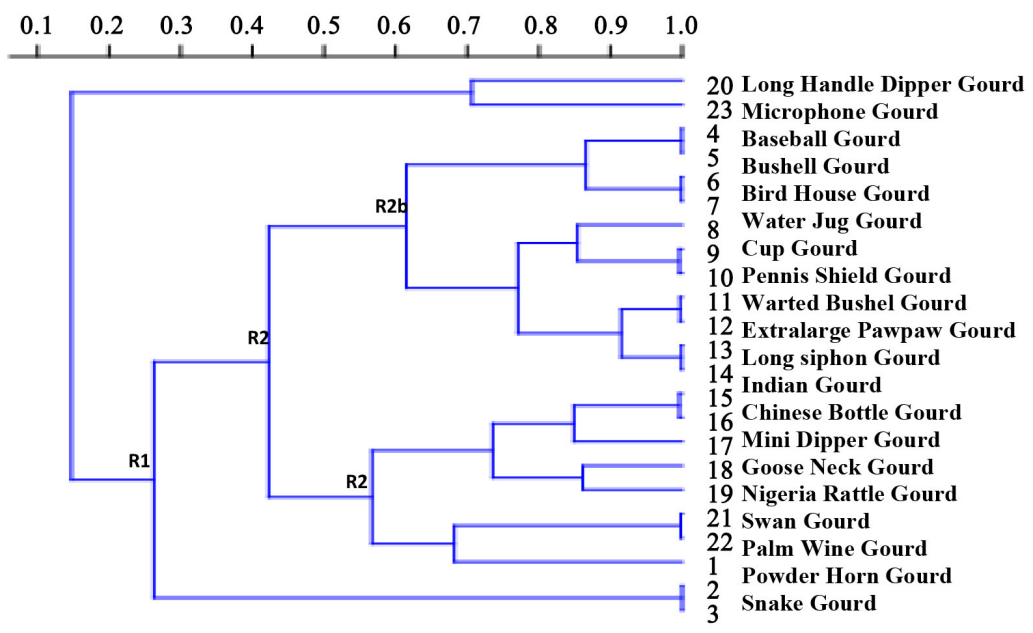

Figure 4. Phylogenetic tree of Lageneria siceraria (Mol.) Standl. 
Table 6. Distance matrix of Lageneria siceraria (Mol.) Standl.

\begin{tabular}{|c|c|c|c|c|c|c|c|c|c|c|c|c|c|c|c|c|c|c|c|c|c|c|c|}
\hline AWALA_A_1F & & 0.02 & 0.02 & 0.02 & 0.02 & 0.02 & 0.02 & 0.02 & 0.02 & 0.02 & 0.02 & 0.02 & 0.02 & 0.02 & 0.02 & 0.02 & 0.02 & 0.02 & 0.02 & 0.02 & 0.02 & 0.02 & 0.02 \\
\hline AWALA_B_RCBL & 0.75 & & 0.01 & 0.02 & 0.02 & 0.02 & 0.02 & 0.02 & 0.02 & 0.02 & 0.02 & 0.02 & 0.02 & 0.02 & 0.02 & 0.02 & 0.02 & 0.02 & 0.02 & 0.02 & 0.02 & 0.02 & 0.02 \\
\hline AWALA_C_1F & 0.76 & 0.04 & & 0.02 & 0.02 & 0.02 & 0.02 & 0.02 & 0.02 & 0.02 & 0.02 & 0.02 & 0.02 & 0.02 & 0.02 & 0.02 & 0.02 & 0.02 & 0.02 & 0.02 & 0.02 & 0.02 & 0.02 \\
\hline AWA & 0.69 & 0.69 & 0.71 & & 0.02 & 0.02 & 0.02 & 0.02 & 0.02 & 0.02 & 0.02 & 0.02 & 0.02 & 0.02 & 0.02 & 0.02 & 0.02 & 0.02 & 0.02 & .02 & .02 & 0.02 & 0.02 \\
\hline AWALA_F_1F & 0.74 & 0.73 & 0.73 & 0.77 & & 0.02 & 0.02 & 0.01 & 0.02 & 0.02 & 0.02 & 0.02 & 0.02 & 0.02 & 0.02 & 0.02 & 0.02 & 0.02 & 0.02 & 0.00 & 0.02 & 0.02 & 0.01 \\
\hline AWALA_G_RCBL & 0.77 & 0.77 & 0.77 & 0.73 & 0.75 & & 0.02 & 0.02 & 0.02 & 0.02 & 0.02 & 0.02 & 0.02 & 0.02 & 0.02 & 0.02 & 0.02 & 0.02 & 0.02 & 0.02 & 0.02 & 0.02 & 0.02 \\
\hline AWALA_H_RCBL & 0.72 & 0.76 & 0.76 & 0.74 & 0.78 & 0.70 & & 0.02 & 0.02 & 0.02 & 0.02 & 0.02 & 0.02 & 0.02 & 0.02 & 0.02 & 0.02 & 0.02 & 0.02 & 0.02 & 0.02 & 0.02 & 0.02 \\
\hline AWALA_I_1F & 0.74 & 0.73 & 0.74 & 0.77 & 0.02 & 0.75 & 0.78 & & 0.02 & 0.02 & 0.02 & 0.02 & 0.02 & 0.02 & 0.02 & 0.02 & 0.02 & 0.02 & 0.02 & 0.01 & 0.02 & 0.02 & 0.01 \\
\hline AWALA_J_RCBL & 0.74 & 0.73 & 0.73 & 0.77 & 0.72 & 0.62 & 0.75 & 0.72 & & 0.02 & 0.02 & 0.02 & 0.02 & 0.02 & 0.02 & 0.02 & 0.02 & 0.02 & 0.02 & 0.02 & 0.02 & 0.02 & 0.02 \\
\hline AWALA_K_1F & 0.77 & 0.72 & 0.73 & 0.73 & 0.70 & 0.76 & 0.75 & 0.70 & 0.76 & & 0.02 & 0.02 & 0.02 & 0.02 & 0.02 & 0.02 & 0.02 & 0.02 & 0.02 & 0.02 & .02 & 0.02 & 0.02 \\
\hline AWALA_L_1F & 0.73 & 0.78 & 0.78 & 0.72 & 0.76 & 0.71 & 0.72 & 0.76 & 0.75 & 0.71 & & 0.02 & 0.02 & 0.02 & 0.02 & 0.02 & 0.01 & 0.02 & 0.02 & 0.02 & 0.02 & 0.02 & 0.02 \\
\hline AWALA_M_1F & 0.72 & 0.74 & 0.75 & 0.78 & 0.72 & 0.77 & 0.76 & 0.73 & 0.71 & 0.77 & 0.69 & & 0.02 & 0.02 & 0.02 & 0.02 & 0.02 & 0.01 & 0.02 & 0.02 & 0.02 & 0.02 & 0.02 \\
\hline AWALA_N_1F & 0.78 & 0.76 & 0.76 & 0.75 & 0.77 & 0.75 & 0.74 & 0.76 & 0.75 & 0.73 & 0.77 & 0.72 & & 0.02 & 0.02 & 0.02 & 0.02 & 0.02 & 0.02 & 0.02 & 0.02 & 0.02 & 0.02 \\
\hline AWALA_O_1F & 0.72 & 0.73 & 0.73 & 0.77 & 0.77 & 0.75 & 0.74 & 0.77 & 0.78 & 0.72 & 0.78 & 0.73 & 0.72 & & 0.02 & 0.02 & 0.02 & 0.02 & 0.02 & 0.02 & 0.02 & 0.02 & 0.02 \\
\hline AWALA_P_1F & 0.76 & 0.74 & 0.75 & 0.72 & 0.72 & 0.74 & 0.73 & 0.72 & 0.78 & 0.73 & 0.71 & 0.73 & 0.75 & 0.76 & & 0.02 & 0.02 & 0.02 & 0.02 & 0.02 & 0.02 & 0.02 & 0.02 \\
\hline AWALA_Q_RCBL & 0.75 & 0.71 & 0.71 & 0.74 & 0.75 & 0.78 & 0.78 & 0.76 & 0.76 & 0.71 & 0.76 & 0.74 & 0.74 & 0.70 & 0.78 & & 0.02 & 0.02 & 0.02 & 0.02 & 0.02 & 0.02 & 0.02 \\
\hline AWALA_R_1F & 0.74 & 0.78 & 0.77 & 0.72 & 0.77 & 0.70 & 0.73 & 0.77 & 0.75 & 0.72 & 0.06 & 0.72 & 0.75 & 0.77 & 0.71 & 0.76 & & 0.02 & 0.02 & 0.02 & 0.02 & 0.02 & 0.02 \\
\hline AWALA_S_1F & 0.72 & 0.74 & 0.75 & 0.78 & 0.72 & 0.76 & 0.76 & 0.73 & 0.71 & 0.76 & 0.70 & 0.03 & 0.71 & 0.73 & 0.73 & 0.74 & 0.72 & & 0.02 & 0.02 & 0.02 & 0.02 & 0.02 \\
\hline AWALA_T_1F & 0.74 & 0.73 & 0.74 & 0.77 & 0.77 & 0.75 & 0.75 & 0.76 & 0.73 & 0.73 & 0.76 & 0.76 & 0.72 & 0.68 & 0.78 & 0.73 & 0.76 & 0.77 & & 0.02 & 0.02 & 0.02 & 0.02 \\
\hline AWALA_U_1F & 0.73 & 0.73 & 0.74 & 0.77 & 0.02 & 0.75 & 0.78 & 0.02 & 0.72 & 0.70 & 0.76 & 0.73 & 0.77 & 0.77 & 0.72 & 0.76 & 0.78 & 0.72 & 0.76 & & 0.02 & 0.02 & 0.01 \\
\hline AWALA_V_1F & 0.78 & 0.78 & 0.78 & 0.74 & 0.71 & 0.72 & 0.77 & 0.72 & 0.75 & 0.76 & 0.72 & 0.76 & 0.77 & 0.75 & 0.72 & 0.72 & 0.72 & 0.76 & 0.70 & 0.72 & & 0.01 & 0.02 \\
\hline AWALA_W_1F & 0.78 & 0.78 & 0.77 & 0.73 & 0.70 & 0.73 & 0.75 & 0.70 & 0.76 & 0.76 & 0.72 & 0.76 & 0.77 & 0.75 & 0.71 & 0.72 & 0.72 & 0.76 & 0.71 & 0.70 & 0.04 & & 0.02 \\
\hline AWALA_X_1F & 0.73 & 0.73 & 0.74 & 0.77 & 0.08 & 0.75 & 0.78 & 0.07 & 0.71 & 0.68 & 0.74 & 0.73 & 0.76 & 0.77 & 0.73 & 0.76 & 0.76 & 0.73 & 0.76 & 0.07 & 0.72 & 0.72 & \\
\hline
\end{tabular}

evolutionary relationship. The quality of the chloroplast DNA was very visible at $850 \mathrm{bp}$ across the landraces of $L$. siceraria using agarose gel electrophoresis (Figure 2; Figure 3). The quantified DNA (Table 3) had an absorbance ratio of 1.2 to 1.8 showing the purity of the DNA, which is in line with the work of [16] [17]. The conserved $r b c L$ gene has been utilized in PCR amplification of chloroplast gene sequences for determining and ratifying phylogenies [18] [19]. The sequence alignment of the twenty-three landraces from Nigeria shows great variation in the arrangement of the nucleotide bases (Figure 4), which is due to gene recombination and mutation.

The sequences were subjected to validation through National Center for Biotechnology Information (NCBI) using Nucleotide Basic Local Alignment Search Tool (BLAST N). The result obtained (Table 4) proves that all the sequences belong to Lagenaria siceraria (Mol.) standl. with percentages ranging from $95 \%$ to $100 \%$ for query cover sequences and $98 \%$ to $100 \%$ for identity sequences with an E-value of 0.00. From the taxonomic report obtained sequence F-Bushel Gourd, K-Warted bushel gourd, U-Long handle dipper, I-Cup gourd, L-Extra-large paw- 
paw gourd, M-Long siphon gourd, S-Swan gourd, T-Palmwine gourd, W-Snake gourd has the highest hits of 44 on Lagenaria siceraria out of 109 total value, sequence O-Chinese gourd, P-Mini dipper gourd and R-Nigeria rattle gourd has the highest hits of 44 on L. siceraria out of the total value of 111 , sequence $\mathrm{V}$ has the highest hits of 44 on Lagenaria siceraria out of 119 total value, sequence C-Caveman club gourd, G-Bird house gourd, H-Water jug gourd, J-Pennis shield gourd, Q-Goose neck gourd obtained the highest hit of 45 out of the total value 104, while sequence A-African bottle gourd, B-Kettle gourd, D-Baseball gourd has a total taxonomic value of 104 out of which 44 were identified as $\mathrm{La}$ genaria siceraria. The total taxonomic value of $\mathrm{N}$-Indian gourd was observed as 110 with the highest hit value being 44 on L. siceraria and sequence X-Microphone gourd finally has a highest value hits of 44 on Lagenaria siceraria out of 105 total value.

Thus the phylogenetic tree (Figure 4) was constructed to show the relatedness of the landraces (biotypes). The Long handle dipper and Microphone gourd are out group from the root, and meets at similarity scale of 0.7 , while Kettle gourd and Caveman club gourd are out group from the second root of the tree meeting at a similarity scale of 1.0. The second root has two main clusters that meets at different similarity scale. The first cluster of the second root (R2a), African bottle gourd is seen as out group at 0.65 while snake gourd and powder horn gourd clustered together at 1.0 similarity scale. Swan gourd and palmwine gourd meets at 0.89 while Mini dipper gourd and Goose neck gourd clustered at 1.0, Nigerian gourd is observed as an out group at 0.85 similarity scale. In the second cluster of the second root (R2b), Cup gourd was an out group at 0.85 while Baseball gourd, Bird house gourd, Water jug gourd, Penis shield gourd, Warted bushel gourd, Extra-large pawpaw gourd, Long siphon gourd, Indian gourd and Chinese gourd met at 1.0 similarity scale. The phytogenetic studies, have therefore aided in proper understanding of the gene pool and genetic variability of the landraces (biotypes) of $L$. siceraria found in Nigeria.

\section{Conclusion}

The use of bioinformatics tools in biosystematics studies of Lagenaria siceraria landraces (biotypes) found in Nigeria has served as a tool in resolving the quagmire in the diversity of $L$. siceraria complex and the classification of the Landraces into proper taxa. Hence molecular systematics of the species demonstrates differences in sequence arrangement that is due to gene recombination and the functional effect of heterologous genes expressed phenotypically on the fruit shape thereby resulting in the diversity of in fruit shapes of Lagenaria siceraria (Mol.) Standl. found in Nigeria.

\section{Conflicts of Interest}

The authors declare no conflicts of interest regarding the publication of this paper. 


\section{References}

[1] Chimonyo, V.G.P. and Modi, A.T. (2013) Seed Performance of Selected Bottle Gourd (Lagenaria siceraria (Molina) Standl.). American Journal of Experimental Agriculture, 3, 740-766. https://doi.org/10.9734/ajea/2013/4114

[2] Clarke, A.C., Burtenshaw, M.K., McLenachan P.A., Erickson, D.L. and Penny, D. (2006) Reconstructing the Origins and Dispersal of the Polynesian Bottle Gourd (Lagenaria siceraria) Molecular Biology and Evolution, 23, 893-900. https://doi.org/10.1093/molbev/msj092

[3] Erickson, D.L., Smith, B.D., Clarke, A.C., Sandweiss, D.H. and Tuross, N. (2004) An Asian Origin for a 10,000 Year Old Domesticated Plant in the Americans. Proceedings of the National Academy of Sciences of the United States of America, 102, 18315-18320. https://doi.org/10.1073/pnas.0509279102

[4] Van Ham, R.C.H.J., Hart, H., Mes, T.H.M. and Sandbrink, J.M. (1994) Molecular Evolution of Noncoding Regions of the Chloroplast Genome in the Crassulaceae and Related Species. Curriculum Genetics, 25, 558-566.

https://doi.org/10.1007/BF00351678

[5] Darwin, C. (1859) The Origin of Species. John Murray, London.

[6] Singh G. (2016) Plant Systematics: An Integrated Approach. Third Edition, Scientific Publishers, India, 157.

[7] Stephen, J.M. (2012) Gourd, Lagenaria siceraria (Molina) Standl. University of Florida, Gainesville, FL, 1.

[8] Zietkiewicz, E., Rafalski, A. and Labuda, D. (1994) Genome Fingerprinting by Simple Sequence Repeat (SSR)-Anchored Polymerase Chain Reaction Amplification. Genomics, 20, 176-183. https://doi.org/10.1006/geno.1994.1151

[9] Wattoo, J.I., Saleem, M.Z., Shahzad, M.S., Arif, A., Hameed, A. and Saleem, M.A. (2016) DNA Barcoding: Amplification and Sequence Analysis of $r b c l$ and mat Kgenome Regions in Three Divergent Plant Species. Advancements in Life Sciences, 4 , 3-7.

[10] Hawtin, G., Iwanaga, M. and Hodgkin, T. (1996) Genetic Resources in Breeding for Adaptation. Euphytica, 92, 255-266. https://doi.org/10.1007/BF00022853

[11] Matzke, M., Kanno, T., Daxinger, L., Huettel, B. and Matzke, A.J.M. (2009) RNA-Mediated Chromatin-Based Silencing in Plants. Targets of RNA-Directed DNA Methylation. Current Opinion in Cell Biology, 21, 367-376. https://doi.org/10.1016/j.ceb.2009.01.025

[12] Afuape, S.O., Okocha, P.I. and Njoku, D. (2011) Multivariate Assessment of the Agromorphological Variability and Yield Components among Sweetpotato (Ipomoea batatas (L.) Lam) Landraces. African Journal of Plant Science, 5,123-132.

[13] Heiser Jr., C.B. (1973) Variation in Bottle Gourd. In: Meggers, B.J., Ayensu, E.S. and Duckworth, W.D., Eds., Tropical Forest Ecosystems in Africa and South America: A Comparative Review, Smithsonian Institution Press, Washington DC, 121-128.

[14] Hollingsworth, P.M., Graham, S.W. and Little, D.P. (2011) Choosing and Using a Plant DNA Barcode. PLoS ONE, 6, 19254-19267.

https://doi.org/10.1371/journal.pone.0019254

[15] Kumar, S., Stecher, G., Li, M., Knyaz, C. and Tamura, K. (2018) MEGA X: Molecular Evolutionary Genetics Analysis across Computing Platforms. Molecular Biology and Evolution, 35, 1547-1549. https://doi.org/10.1093/molbev/msy096

[16] Glasel, J. (1995) Validity of Nucleic Acid Purities Monitored by 260/280 Absorbance Ratios. BioTechniques, 18, 62-63. 
[17] Sambrook, J. and Russell, D. (2001) Molecular Cloning: A Laboratory Manual. 3rd Edition, Cold Spring Harbor Laboratory Press, New York.

[18] Ronsted, N., Law, S., Thornton, H., Fay, M.F. and Chase, M.W. (2005) Molecular Phylogenetic Evidence for the Monophyly of Fritillariaand Lilium (Liliaceae; Liliales) and the Infrageneric Classification of Fritillaria. Molecular Phylogenetics and Evolution, 35, 509-527. https://doi.org/10.1016/j.ympev.2004.12.023

[19] Türktas, M., Aslay, M., Kaya, E. and Ertugrul, F. (2012) Molecular Characterization of Phylogenetic Relationships in Fritillaria Species Inferred from Chloroplast trnL-trnF Sequences. Turkish Journal of Biology, 36, 552-560. 